\title{
Malliavin calculus of Bismut type without probability
}

\author{
RÉMI LÉANDRE \\ Institut de Mathématiques, Université de Bourgogne, 21000 Dijon, France \\ R.C.T.P. Central Visayan Institute, Jagna, Bohol, Philippines \\ E-mail: Remi.leandre@u-bourgogne.fr \\ Dedicated to Professor Sinha
}

Abstract. We translate in semigroup theory Bismut's way of the Malliavin calculus.

Keywords. Malliavin calculus without probability.

\section{Introduction}

There are many types of infinite dimensional analysis (see works of Hida, Fomin, Albeverio, Elworthy, Berezanskii, etc) but one of the specificities of Malliavin calculus is that it can be applied to diffusions. Namely, one of the specificity of Malliavin calculus is to complete the classical differential operations on the Wiener space, such that functionals which belong to all the Sobolev spaces of Malliavin calculus are in general only almost surely defined, because there is no Sobolev imbedding theorem in infinite dimension. Diffusions, almost surely defined, belong to all the Sobolev spaces of Malliavin calculus.

By using this functional analysis approach, Malliavin $\left[\mathrm{Ma}_{1}\right]$ got a probabilistic proof of Hoermander's theorem (see $\left[\mathrm{IW}|\mathrm{Me}| \mathrm{St}\left|\mathrm{Ma}_{1}\right| \mathrm{Ma}_{2} \mid \mathrm{Nu}\right]$ for a pedagogical introduction).

Bismut has avoided the heavy apparatus of functional analysis of Malliavin calculus, in order to prove again Hoermander's theorem by probabilistic methods $\left[\mathrm{B}_{1}[\mathrm{No}]\right.$.

We show that Bismut's mechanism can be suitably interpreted in terms of semigroup theory. We avoid using probability theory, but this work is the translation in semigroup theory of the work of Bismut. Remarkable formulas can be seen with the intuition of probability. Let us remark that it is not the first case that probability can explain easily magical formulas: the mysterious rescaling of Getzler's proof of the Index theorem can be easily interpreted in terms of probability theory in the work of Léandre $\left[\mathrm{L}_{3}\right]$. We refer to the survey of Léandre $\left[\mathrm{L}_{2}\right]$ for various probabilistic proofs of the Index theorem, including Bismut proof $\left[\mathrm{B}_{2}\right]$.

For the sake of simplicity, we work in the elliptic case because in this case, the method of Léandre $\left[\overline{\left.\mathrm{L}_{1} \mid \mathrm{L}_{4}\right]}\right.$ of inversion of the Malliavin matrix can be easily interpreted in terms of semigroup theory.

We use the classical results of differentiability of the solutions of parabolic equations which depend on a parameter, which are coming in stochastic analysis from the stochastic flow theorem. 


\section{Cameron-Martin-Girsanov-Maruyama formula in semigroup theory}

Let us consider some vector fields $X_{i}, i=0, \ldots, m$ on $R^{d}$ with bounded derivatives of all order. Let $L$ be the Hoermander type operator

$$
L f=X_{0}+1 / 2 \sum_{i>0} X_{i}^{2}
$$

acting on smooth bounded functions on $R^{d}$. It can be written as

$$
L f=\left\langle X_{0}, D f\right\rangle+1 / 2 \sum_{i>0}\left\langle D X_{i} X_{i}, D f\right\rangle+1 / 2 \sum_{i>0}\left\langle X_{i}, D^{2} f, X_{i}\right\rangle .
$$

In (2.1), vector fields are considered as first order differential operators and in (2.2) vectors fields are considered as smooth applications from $R^{d}$ into $R^{d}$. Let us consider the generator

$$
L^{h}=L+\sum_{i>0} h_{t}^{i} X_{i}
$$

where $t \rightarrow h_{t}^{i}$ are smooth bounded functions which do not depend on $x . L^{h}$ generates an inhomogeneous Markov semigroup $P^{h}$ acting on bounded continuous functions on $R^{d}$.

Let us consider on $R^{d+1}$ some vector fields

$$
\tilde{X}_{i}^{t}=\left(X_{i}, h_{t}^{i} u\right)
$$

and the generator acting on smooth functions of $\tilde{f}$ on $R^{d+1}$ :

$$
\begin{aligned}
\tilde{L}^{h}(\tilde{f})= & \left\langle X_{0}, \tilde{D} \tilde{f}\right\rangle+1 / 2 \sum_{i>0}\left\langle D X_{i} X_{i}, \tilde{D} \tilde{f}\right\rangle \\
& +1 / 2 \sum_{i>0}\left\langle\tilde{X}_{i}^{t}, \tilde{D}^{2} \tilde{f}, \tilde{X}_{i}^{t}\right\rangle .
\end{aligned}
$$

It generates a semigroup $\tilde{P}^{h}$ operating on the bounded continuous functions on $R^{d+1}$. In the sequel, for the integrability conditions, we refer to the Appendix.

\section{Theorem 2.1 (Quasi-invariance).}

$$
P_{t}^{h} f(x)=\tilde{P}_{t}^{h}[u f](x, 1) .
$$

Proof. Since the vector fields $\tilde{X}_{i}^{t}$ are linear in $u$, we have

$$
\tilde{P}^{h}[u f]\left(x, u_{0}\right)=\tilde{P}^{h}[u f](x, 1) u_{0}
$$

for any bounded continuous $f$ on $R^{d}$ such that

$$
\tilde{L}^{h} \tilde{P}^{h}[u f](\cdot, \cdot)\left|(x, 1)=L^{h} \tilde{P}^{h}[u f](\cdot, 1)\right|(x) .
$$

Therefore the result arises by using uniqueness of the solution of the parabolic equation associated to $L^{h}$.

Remark. In order to see from where this remarkable formula comes, we use the stochastic analysis. Let $w_{t}^{i}$ be $m$ Brownian motions and let $Y=X_{0}+1 / 2 \sum_{i>0} D X_{i} X_{i}$ the vector 
field on $R^{d}$. We consider the following stochastic differential equation in Itô sense on $R^{d}$ starting from $x$ :

$$
\delta x_{t}^{h}(x)=Y\left(x_{t}^{h}(x)\right) \mathrm{d} t+\sum_{i>0} h_{t}^{i} X_{i}\left(x_{t}^{h}(x)\right) \mathrm{d} t+\sum_{i>0} X_{i}\left(x_{t}^{h}(x)\right) \delta w_{t}^{i} .
$$

It is a classical result of stochastic analysis that:

$$
P_{t}^{h} f(x)=E\left[f\left(x_{t}^{h}(x)\right)\right] \text {. }
$$

On the other hand, let us consider the Itô equation on $R^{d+1}$ starting from $(x, 1)$ :

$$
\delta \tilde{x}_{t}(x)=Y\left(\tilde{x}_{t}(x) \mathrm{d} t+\sum \tilde{X}_{i}^{t}\left(\tilde{x}_{t}(x)\right) \delta w_{t}^{i} .\right.
$$

We have

$$
\tilde{P}_{t}^{h}[F](x, 1)=E\left[F\left(\tilde{x}_{t}(x)\right)\right]
$$

if $F$ is a bounded continuous function on $R^{d+1}$. Moreover, the classical Girsanov formula shows us that, by using stochastic calculus we have

$$
E\left[f\left(x_{t}^{h}(x)\right)\right]=E\left[F\left(\tilde{x}_{t}(x)\right)\right],
$$

where $F(x, u)=u f(x)$.

Let us consider the vector field $\bar{X}_{i}^{h}=\left(X_{i}, h_{t}^{i}\right)$ and the generator on $R^{d+1}$ acting on smooth functions $\tilde{f}$ on $R^{d+1}$ :

$$
\bar{L}^{h} \tilde{f}=\left\langle X_{0}, \tilde{D} f\right\rangle+1 / 2 \sum_{i>1}\left\langle D X_{i} X_{i}, \tilde{D} \tilde{f}\right\rangle+1 / 2 \sum_{i>0}\left\langle\bar{X}_{i}^{h}, \tilde{D}^{2} \tilde{f}, \bar{X}_{i}^{h}\right\rangle .
$$

It generates a semigroup $\bar{P}^{h}$ acting on the bounded continuous functions on $R^{d+1}$.

Theorem 2.2 (Elementary integration by parts formula).

$$
\int_{0}^{t} P_{t-s} \sum_{i>0} h_{u}^{i} X_{i}\left[P_{s} f\right] \mathrm{d} s=\bar{P}_{t}^{h}[u f](x, 0) .
$$

Proof. We have $\bar{P}_{t}^{h}[u f]\left(x, u_{0}\right)=A_{t}(x) u_{0}+B_{t}(x)$ because $\partial / \partial u$ commute with $\bar{L}^{h}$. Therefore,

$$
\bar{P}_{t}^{h}[u f]\left(x, u_{0}\right)=P_{t}[f](x) u_{0}+\bar{P}_{t}^{h}[u f](x, 0)
$$

such that

$$
\frac{\partial}{\partial t} \bar{P}^{h}[u f](\cdot, \cdot)\left|(x, 0)=L \bar{P}^{h}[u f](\cdot, 0)\right|(x)+\sum_{i \geq 1} h_{t}^{i}\left\langle X_{i}, P_{t}[f](x)\right\rangle
$$

with starting condition 0 .

On the other hand, $F(t, x)=\int_{0}^{t} P_{t-s}\left[\sum_{i>0} h_{s}^{i} X_{i}\left[P_{s}[f]\right]\right] \mathrm{d} s$ is a solution of the parabolic equation:

$$
\frac{\partial}{\partial t} F(t, x)=L F(t, x)+\sum_{i>0} h_{t}^{i} X_{i} P_{t}[f](x)
$$

with starting condition 0 . The result arises by unicity of the solution of this parabolic equation. 
Remark. Let us show from where this formula comes by stochastic analysis. In (2.9), we put a small $\lambda$ before $h_{t}^{i}$ and we get two processes $x_{t}^{\lambda}(x)$ and $\tilde{x}_{t}^{\lambda}(x)$. We get the formula:

$$
E\left[D f\left(x_{t}^{0}\right) \frac{\partial}{\partial \lambda} x_{t}^{0}(x)\right]=E\left[D F\left(\tilde{x}_{t}^{0}\right) \frac{\partial}{\partial \lambda} \tilde{x}_{t}^{0}(x)\right] .
$$

We recognize in this last expression the quantity

$$
f\left(x_{t}^{0}(x)\right) \int_{0}^{t} \sum_{i>0} h_{t}^{i} \delta w_{t}^{i}
$$

which gives the second term of (2.15). On the other hand, $\frac{\partial}{\partial \lambda} x_{t}^{0}(x)$ is a solution of the stochastic differential Stratonovitch equation:

$$
\begin{aligned}
\mathrm{d} \frac{\partial}{\partial \lambda} x_{t}(x)= & D X_{0}\left(x_{t}^{0}\right) \frac{\partial}{\partial \lambda} x_{t}(x) \mathrm{d} t+\sum D X_{i}\left(x_{t}(x)\right) \frac{\partial}{\partial \lambda} x_{t}(x) \mathrm{d} w_{t}^{i} \\
& +\sum h_{t}^{i} X_{i}\left(x_{t}^{0}(x)\right) \mathrm{d} t
\end{aligned}
$$

starting from 0 . It can be solved by the method of variation of constant. Let $U_{t}$ be the solution of the matricial equation starting from $I$ :

$$
\mathrm{d} U_{t}=D X_{0}\left(x_{t}^{0}(x)\right) U_{t} \mathrm{~d} t+\sum D X_{i}\left(x_{t}^{0}(x)\right) U_{t} \mathrm{~d} w_{t}^{i} .
$$

It is a classical result of stochastic analysis that:

$$
\frac{\partial}{\partial \lambda} x_{t}^{0}(x)=U_{t} \int_{0}^{t} U_{s}^{-1} h_{s}^{i} X_{i}\left(x_{s}^{0}(x)\right) \mathrm{d} s .
$$

But (see Lemma 3.2 below), we have

$$
\sum_{i>0} h_{t}^{i} X_{i} P_{t}[f](x)=\sum_{i>0} E\left[D f\left(x_{t}^{0}(x)\right) U_{t}\right](x, I) X_{i}(x) .
$$

The result follows by doing the change of variable $s \rightarrow t-s$.

\section{Malliavin's theorem in semigroup theory}

For the integrability condition, we refer to the appendix. Let us consider the vector fields on $R^{d} \times G l\left(R^{d}\right) \times M^{d}=V^{d}$ where $G l\left(R^{d}\right)$ is the space of invertible matrices on $R^{d}$, and $M^{d}$ the space of matrices on $R^{d}$ :

$$
\hat{X}_{i}=\left(X_{i}, D X_{i} U, 0\right)
$$

and

$$
\hat{X}=\left(0,0, \sum_{i>0}\left\langle U^{-1} X_{i}, \cdot\right\rangle^{2}\right) .
$$

Let us consider the semigroup operating on continuous bounded functionals on $V^{d}$ generated by $\hat{L}$ :

$$
\begin{aligned}
\hat{L} \hat{f}= & 1 / 2 \sum_{i>0}\left\langle\hat{X}_{i}, \hat{D}^{2} \hat{f}, \hat{X}_{i}\right\rangle \\
& +1 / 2 \sum_{i>0}\left\langle D \hat{X}_{i} \hat{X}_{i}, \hat{D} \hat{f}\right\rangle+\left\langle\hat{X}_{0}, \hat{D} \hat{f}\right\rangle+\langle\hat{X}, \hat{D} \hat{f}\rangle .
\end{aligned}
$$


We consider $\hat{P}_{t}$ the semigroup associated to $\hat{L}$. It is associated to the system of stochastic differential equation (in Stratonovitch form):

$$
\begin{aligned}
\mathrm{d} x_{t}(x) & =X_{0}\left(x_{t}(x)\right) \mathrm{d} t+\sum_{i>0} X_{i}\left(x_{t}(x)\right) \mathrm{d} w_{t}^{i} \\
\mathrm{~d} U_{t}(x) & =D X_{0}\left(x_{t}(x)\right) U_{t} \mathrm{~d} t+\sum_{i>0} D x_{i}\left(x_{t}(x)\right) U_{t} \mathrm{~d} w_{t}^{i} \\
\mathrm{~d} V_{t} & =\sum_{i>0}\left\langle U_{t}^{-1} X_{i}\left(x_{t}(x), \cdot\right\rangle^{2}\right.
\end{aligned}
$$

starting from $(x, U, V)$. If we consider $U=I d$ and $V=0, U_{t}$ corresponds to $D x_{t}(x)$ and $V_{t}$ to the so-called Malliavin matrix.

Theorem 3.1 $\left[\mathrm{Ma}_{\mathbf{1}}, \mathbf{M a}_{2}\right]$. If $\hat{P}_{t}\left[V^{-p}\right](x, I, 0)<\infty$ for all $p, P_{t} f(x)=\int_{R^{d}} p_{t}(x, y) f(y) \mathrm{d} y$ where $y \rightarrow p_{t}(x, y)$ is smooth positive.

Let us study $X_{i} P_{t}[f]$.

Lemma 3.2. Let $\bar{P}_{t}$ be the semigroup acting on bounded continuous functions on $R^{d} \times$ $G l\left(R^{d}\right)$ associated to $\bar{L}=\bar{X}_{0}+1 / 2 \sum \bar{X}_{i}^{2}$ where $\bar{X}_{i}=\left(X_{i}, D X_{i} U\right)$. We get

$$
D P_{t}[f](x)=\bar{P}_{t}[D f U](x, I) .
$$

Proof. We write

$$
\psi_{t}\left(x, U_{0}\right)=\bar{P}_{t}[D f U]\left(x, U_{0}\right)=\bar{P}_{t}\left[D f U U_{0}\right](x, I) .
$$

In $U_{0}=I$, we can compute $\bar{L} \psi_{t}$. We get

$$
\begin{aligned}
& 1 / 2 \sum_{i>0}\left\langle\bar{X}_{i}, v \bar{D}^{2} \psi_{t}, \bar{X}_{i}\right\rangle+1 / 2 \sum_{i>0}\left\langle\overline{D X}_{i} \bar{X}_{i}, \bar{D} \psi_{t}\right\rangle+\left\langle\bar{X}_{0}, \bar{D} \psi_{t}\right\rangle \\
& =1 / 2 \sum_{i>0}\left\langle X_{i}, D^{2} \psi_{t}, X_{i}\right\rangle+1 / 2 \sum_{i>0}\left\langle D X_{i} X_{i}, D \psi_{t}\right\rangle \\
& \quad+\sum_{i>0}\left\langle X_{i}, D \psi_{t}, D X_{i}\right\rangle+1 / 2 \sum\left\langle D X_{i} D X_{i}, \psi_{t}\right\rangle+\sum_{i>0}\left\langle D^{2} X_{i} X_{i}, \psi_{t}\right\rangle \\
& \quad+\left\langle X_{0}, D \psi_{t}\right\rangle+\left\langle D X_{0}, \psi_{t}\right\rangle
\end{aligned}
$$

such that

$$
\begin{aligned}
\frac{\partial}{\partial t} \psi_{t}(x, I)= & 1 / 2 \sum\left\langle X_{i}, D^{2} \psi_{t}, X_{i}\right\rangle+1 / 2 \sum\left\langle D X_{i} X_{i}, D \psi_{t}\right\rangle \\
& +1 / 2 \sum\left\langle X_{i}, D \psi_{t}, D X_{i}\right\rangle+1 / 2 \sum\left\langle D X_{i} D X_{i}, \psi_{t}\right\rangle \\
& +1 / 2 \sum\left\langle D^{2} X_{i} X_{i}, \psi_{t}\right\rangle+\left\langle X_{0}, D \psi_{t}\right\rangle+\left\langle D X_{0}, \psi_{t}\right\rangle
\end{aligned}
$$

with initial condition $D f$.

Moreover, $P_{t} f$ satisfies the equation

$$
\frac{\partial}{\partial t} P_{t} f=1 / 2 \sum_{i>0}\left\langle X_{i}, D^{2} P_{t} f, X_{i}\right\rangle+1 / 2 \sum_{i>0}\left\langle D X_{i} X_{i}, D P_{t} f\right\rangle+\left\langle X_{0}, D P_{t} f\right\rangle
$$

such that $\frac{\partial}{\partial t} D P_{t} f$ satisfies (3.7) with the same initial condition $D f$. Therefore we get the result by the unicity of the solution of (3.7). 
Remark. Let us show from where this remarkable formula comes. We have

$$
P_{t} f(x),=E\left[f\left(x_{t}(x)\right)\right]
$$

such that

$$
D P_{t} f(x)=E\left[D f\left(x_{t}(x)\right) D x_{t}(x)\right]
$$

This result arises by the considerations following (3.4).

Let us proceed as in [No]. We define on $R^{d_{1}} \times \cdots \times R^{d_{k}}$ some vector fields

$$
X_{i}^{\mathrm{tot}}=\left(X_{i}^{1}\left(x_{1}\right), \ldots, X_{i}^{j}\left(x^{1}, \ldots, x^{j}\right), \ldots, X_{i}^{k}\left(x^{1}, \ldots, x^{k}\right)\right)
$$

where

$$
\begin{aligned}
X_{i}^{k}\left(x^{1}, \ldots, x^{k}\right)= & X_{1, i}^{k}\left(x^{1}, \ldots, x^{k-1}\right) x^{k} \frac{\partial}{\partial x^{k}}+X_{2, i}^{k}\left(x^{1}, \ldots, x^{k}\right) \\
& +X_{3, i}^{k}\left(x^{1}, \ldots, x^{k-1}\right)
\end{aligned}
$$

where $X_{1, i}^{k}$ has bounded derivatives of all orders, $X_{2, i}^{k}=X_{2, i}^{k}\left(x^{1}, \ldots, x^{k-1}\right) \frac{\partial}{\partial x_{k}}$ has derivatives of all orders with polynomial growth and $X_{3, i}^{k}$ has derivatives with polynomial growths.

We can define a semigroup $P^{\mathrm{tot}, k}$ associated to $1 / 2 \sum_{i>0}\left(X_{i}^{\mathrm{tot}, k}\right)^{2}+X_{0}^{\mathrm{tot}, k}$. We get if $k^{\prime}<$ $k$,

$$
P^{\mathrm{tot}, k}\left[f^{\mathrm{tot}, k^{\prime}}\right]\left(x^{\mathrm{tot}, k}\right)=P^{\mathrm{tot}, k^{\prime}}\left[f^{\mathrm{tot}, k^{\prime}}\right]\left(x^{\mathrm{tot}, k^{\prime}}\right) .
$$

$x^{k}$ has to be seen as a matrix if $X_{1, i}^{k}$ is not equal to zero.

Equation (3.14) can be seen by using stochastic analysis because $P^{\text {tot }, k}$ is associated to a step by step system of stochastic differential equations. Moreover, for all $p$ we get as follows.

PROPOSITION 3.3.

$$
\begin{gathered}
P^{\mathrm{tot}, k}\left(\left|x^{k}\right|^{p}\right]<\infty \text { and if } X_{2, i}^{k}\left(x^{1}, \ldots, x^{k}\right)=0=X_{3, i}^{k}\left(x^{1}, \ldots, x^{k-1}\right), \\
P^{\mathrm{tot}, k}\left(\left|\left(x^{k}\right)^{-1}\right|^{p}\right]\left(x^{1}, \ldots, x^{k-1}, I\right)<\infty
\end{gathered}
$$

We refer to the Appendix for the proof of this proposition.

Instead of considering the generator $X_{0}+1 / 2 \sum_{i>0} X_{i}^{2}$ by $\sum X_{i} h_{t}^{i}$ where $h_{t}^{i}$ is deterministic, we consider the perturbation by $\langle\phi(x), h\rangle^{i} X_{i}$ where $\phi$ is smooth bounded with derivatives of polynomial growths.

We get a semigroup $P_{t}^{\lambda}$ associated to the generator $X_{0}+1 / 2 \sum X_{i}^{2}+\lambda \sum_{i>0}\langle\phi(x), h\rangle^{i} X_{i}$.

Lemma 3.4. Let $\bar{X}_{i}=\left(X_{i}, D X_{i} U\right), i=0,1, \ldots, m$ and $\tilde{X}_{0}=\left(0, \sum X_{i}\left\langle\phi(x), h_{t}^{i}\right\rangle\right)$ and $\bar{P}_{t}^{\prime}$ be the semigroup associated to $1 / 2 \sum_{i>0} \bar{X}_{i}^{2}+\bar{X}_{0}+\tilde{X}_{0}$. We get

$$
\frac{\partial}{\partial \lambda} P_{t}^{0}[f](x)=\bar{P}_{t}^{\prime}[D f U](x, 0) .
$$


Proof. The integrability conditions are satisfied by Proposition 3.4.

Let $\bar{P}_{t}$ be the semigroup associated to $1 / 2 \sum \bar{X}_{i}^{2}+\bar{X}_{0}$. If the Volterra expansion converges for the $C^{k}$ uniform norm on each compact, we get

$$
\begin{aligned}
& \bar{P}_{t}^{\prime}[D f U](x, 0) \\
& \quad=\sum(-1)^{n} \int_{0<s_{1}<\cdots<s_{n}<t} \bar{P}_{s_{1}} \tilde{X}_{0} \ldots \tilde{X}_{0} \bar{P}_{t-s_{n}}[D f U](x, 0) \mathrm{d} s_{1} \ldots \mathrm{d} s_{n} .
\end{aligned}
$$

But $\bar{P}_{t}[D f U]\left(x, U_{0}\right)$ is linear in $U_{0}$ and $\tilde{X}_{0} U_{0}=\sum X_{i}\left\langle\phi(x), h_{t}\right\rangle^{i}=Y_{i}$ which does not depend on $U_{0}$. We deduce that

$$
\bar{P}_{t}^{\prime}[D f U](x, 0)=-\int_{0}^{t} P_{s_{1}} \sum_{i>0} Y_{i} \bar{P}_{t-s_{1}}[D f U](x, I)
$$

which is the formula of Lemma 3.2.

Remark. Let us show from where this formula comes. $P_{t}^{\lambda}$ is associated to the stochastic differential equation in the Stratonovitch sense:

$$
\mathrm{d} x_{t}^{\lambda}=X_{0}\left(x_{t}^{\lambda}\right) \mathrm{d} t+\sum_{i>0} X_{i}\left(x_{t}^{\lambda}\right) \mathrm{d} w_{t}^{i}+\lambda \sum_{i>0} X_{i}\left(x_{t}^{\lambda}\right)\left\langle\phi\left(x_{t}^{\lambda}\right), h_{t}^{i}\right\rangle \mathrm{d} t
$$

such that

$$
\frac{\partial}{\partial \lambda} P_{t}^{0}[f](x)=E\left[D f\left(x_{t}^{0}(s) \frac{\partial}{\partial \lambda} x_{t}^{0}(x)\right]\right.
$$

But $\frac{\partial}{\partial \lambda} x_{t}^{0}$ satisfies the stochastic differential equation in the Stratonovitch sense starting from 0:

$$
\begin{aligned}
\mathrm{d} \frac{\partial}{\partial \lambda} x_{t}^{0}= & D X_{0}\left(x_{t}^{0}\right) \frac{\partial}{\partial \lambda} x_{t}^{0}+\sum_{i>0} D X_{i}\left(x_{t}^{0}\right) \frac{\partial}{\partial \lambda} x_{t}^{0} \mathrm{~d} w_{t}^{i} \\
& +\sum_{i>0} X_{i}\left(x_{t}^{0}\right)\left\langle\phi\left(x_{t}^{0}\right), h_{t}^{i}\right\rangle \mathrm{d} t
\end{aligned}
$$

and the couple of $\left(x_{t}^{0}, \frac{\partial}{\partial \lambda} x_{t}^{0}\right)$ is associated to $\bar{P}_{t}^{\prime}$. 
PROPOSITION 3.5. [B]

$$
\bar{P}_{t}^{\prime}[D f U](x, 0)=Q_{t}[f u](x, 0),
$$

where $Q_{t}$ is the semigroup generated by

$$
\tilde{L} \tilde{f}=1 / 2 \sum_{i>0}\left\langle\tilde{X}_{i}, \tilde{D}^{2} \tilde{f}, \tilde{X}_{i}\right\rangle+1 / 2 \sum_{i>0}\left\langle D X_{i} X_{i}, \tilde{D} \tilde{f}\right\rangle+\left\langle X_{0}, \tilde{D} \tilde{f}\right\rangle
$$

on $R^{d+1}$ where

$$
\tilde{X}_{i}=\left(X_{i},\langle\phi(x), h\rangle^{i}\right) .
$$

We get the following.

Proof. We remark that the vector fields involved with $\tilde{L}$ commute with $\partial / \partial u$ such that $Q_{t}[f u]\left(x, u_{0}\right)=A(x) u_{0}+B(x)$. We remark therefore that

$$
Q_{t}[f u]\left(x, u_{0}\right)=Q_{t}[f u](x, 0)+P_{t}[f](x) u_{0}
$$

such that

$$
\frac{\partial}{\partial t} Q_{t}[f u](x, 0)=L Q_{t}[f u](x, 0)+\sum Y_{i} P_{t}[f](x) .
$$

Therefore the result is as in the proof of Theorem 2.2.

In the previous formula, $\bar{P}_{t}^{\prime}[D f U](x, 0)$ is a scalar. We would like to get a vector. In Lemma 3.4, we choose $\tilde{X}_{0}=X_{i}^{t}\left(U^{-1} X_{i}\right)$ where $U$ is chosen according to Lemma 3.2. We get with this extension

$$
\bar{P}_{t}^{\prime \prime}[D f V](x, 0)=Q_{t}[f u](x, I, 0),
$$

where $u$ is a vector in $Q_{t}[f u](x, I, 0)$ (see Lemma 3.7 for the definition of $\bar{P}^{\prime \prime}$ ).

Lemma 3.6. If $\bar{P}_{t}^{\prime \prime}\left[\left|V^{-1}\right| p\right](x, I, 0)$ is finite for all $p, f \rightarrow P_{t}[f](x)$ has a smooth density.

Proof. We can use Proposition 3.5 to the extended semigroup of Proposition 3.3 where we replace $\phi(x)$ by ${ }^{t}\left(U^{-1} X_{i}\right)$. We apply Proposition 3.5 to $(V, f) \rightarrow f V^{-1}$. We get

$$
\bar{P}_{t}^{\prime \prime}\left[D\left(f V^{-1}\right) V\right](x, 0)=P_{t}[D f](x)+\text { terms. }
$$

We iterate this procedure. We get if $\bar{P}_{t}^{\prime \prime}\left[\left|V^{-1}\right| p\right](x, I, 0)<\infty$ for all $p$ that

$$
P_{t}\left[D^{r} f\right](x) \leq C_{r}\|f\|_{\infty}
$$

for all $r$ for the supremum norm $\|\cdot\|_{\infty}$ on functions on $R^{d}$. Therefore the result.

Proof of Malliavin's theorem. We have if $\hat{P}_{t}\left[\left|V^{-1}\right| p\right](x, I, 0)<\infty$ for all $p$ that $\bar{P}_{t}^{\prime \prime}\left[\left|V^{-1}\right|^{p}\right](x, I, 0)<$ $\infty$ for all $p$. For that, we use the following lemma.

Lemma 3.7. Let $\tilde{X}_{0}^{\prime \prime},=(0,0, Y)$ where $Y$ depends on the previous variables and has derivatives with polynomial growth. Let $\tilde{X}_{i}=\left(X_{i}, D X_{i} U, D X_{i} V\right)$. Let $\bar{P}_{t}^{\prime \prime}$ be the semi-group associated with $1 / 2 \sum \tilde{X}_{i}^{2}+\tilde{X}_{0}+\tilde{X}_{0}^{\prime \prime}$. We have if $f$ is a homogeneous polynomial in $V$, $\bar{P}_{t}^{\prime \prime}[f]\left(x, I, V_{0}\right)=\hat{P}_{t}\left[f\left(U V+U V_{0}\right)\right](x, I, 0)$ where we have replaced for $\hat{P}_{t}\left\langle U^{-1} X_{i},\right\rangle^{2}$ by $U^{-1} Y$ in (3.2). 
Proof. Let $\tilde{P}_{t}$ be the semigroup associated to $1 / 2 \sum \tilde{X}_{i}^{2}+\tilde{X}_{0}$. It transforms a homogeneous polynomial in $V$ into a homogeneous polynomial in $V$ of same order (see (3.32)). Therefore, we have

$$
\begin{aligned}
& \bar{P}_{t}^{\prime \prime}[f]\left(x, I, V_{0}\right) \\
& \quad=\sum(-1)^{n} \int_{0<s_{1}<\cdots<s_{n}<t} \tilde{P}_{s_{1}} Y \tilde{P}_{s_{2}-s_{1}} Y \ldots \tilde{P}_{t-s_{n}}[f]\left(x, I, V_{0}\right) .
\end{aligned}
$$

We put

$$
\int_{0<s_{1}<\cdots<s_{n}<t} \tilde{P}_{s_{1}} Y \tilde{P}_{s_{2}-s_{1}} Y \ldots \tilde{P}_{t-s_{n}}[f]\left(x, I, V_{0}\right)=I_{n}\left(s_{1}, \ldots, s_{n}\right) .
$$

Let us recall that $\hat{P}_{t}$ is a Markov semigroup. Let $\hat{E}^{s_{1}, \ldots, s_{n}, 1}$ be the law of $x_{s_{1}}, U_{s_{1}}, \ldots, x_{s_{n}}$, $U_{s_{n}}, x_{1}, U_{1}$, starting from $(x, I)$ according this semigroup. We recognize in $I_{n}\left(s_{1}, \ldots, s_{n}\right)$,

$\hat{E}^{s_{1}, \ldots, s_{n}, 1}\left[D^{n} f\left(U_{1} V_{0}\right) U_{1} U_{s_{1}}^{-1} Y\left(x_{s-1}, U_{s_{1}}\right) U_{1} U_{s_{2}}^{-1} Y\left(x_{s_{2}}, U_{s_{2}}\right) \cdots U_{1} U_{s_{n}}^{-1} Y\left(x_{s_{n}}, U_{s_{n}}\right)\right]$.

Therefore the series (3.30) is finite and (3.30) is valid. But this last expression is nothing else but

$$
\bar{P}_{s_{1}} \hat{X}_{0} \bar{P}_{s_{2}-s_{1}} \cdots \hat{X}_{0} \bar{P}_{t-s_{n}}\left[f\left(\cdot, \cdot\left(V+V_{0}\right)\right)\right](x, I, 0) .
$$

Therefore the series (3.30) is equal to

$$
\begin{aligned}
& \sum(-1)^{n} \int_{0<s_{1}<\cdots<s_{n}<t} \bar{P}_{s_{1}} \hat{X}_{0} \bar{P}_{s_{2}-s_{1}} \cdots \hat{X}_{0} \bar{P}_{t-s_{n}}\left[f\left(\cdot, \cdot\left(V+V_{0}\right)\right)\right](x, I, 0) \\
& \quad=\hat{P}_{t}\left[f\left(\cdot, \cdot\left(V+V_{0}\right)\right)\right](x, I, 0) .
\end{aligned}
$$

Therefore the result.

Remark. Let us show from where this formula comes. $\bar{P}_{t}^{\prime \prime}$ is associated to the system of stochastic Stratonovitch differential equation,

$$
\begin{aligned}
\mathrm{d} x_{t}(x) & =X_{0}\left(x_{t}(x) \mathrm{d} t+\sum_{i>0} X_{i}\left(x_{t}\right) \mathrm{d} w_{t}^{i},\right. \\
\mathrm{d} U_{t} & =D X_{0}\left(x_{t}(x)\right) U_{t} \mathrm{~d} t+\sum_{i>0} D X_{i}\left(x_{t}(x)\right) U_{t} \mathrm{~d} w_{t}^{i}, \\
\mathrm{~d} V_{t} & =D X_{0}\left(x_{t}(x)\right) V_{t} \mathrm{~d} t+\sum_{i>0} D X_{i}\left(x_{t}(x)\right) V_{t} \mathrm{~d} w_{t}^{i}+Y\left(x_{t}(x), U_{t}\right) \mathrm{d} t
\end{aligned}
$$

starting from $\left(x, I, V_{0}\right)$. We can solve the last equation by the method of the variation of constant, and we find that

$$
V_{t}=U_{t}\left(V_{0}+\int_{0}^{t} U_{s}^{-1} Y\left(x_{s}(x), U_{s}\right) \mathrm{d} s\right) .
$$

Therefore the result. 


\section{Inversion of the Malliavin matrix in semigroup theory}

In Theorem 3.1, $V$ is called Malliavin's covariance quadratic form. To simplify this work, we will do the following elliptic hypothesis assumption in $x$ :

$$
\sum_{i>1}\left\langle X_{i}(x), \xi\right\rangle^{2} \geq C|\xi|^{2}
$$

for some $C>0$.

Lemma 4.1. If $|\xi|=1$,

$$
\hat{P}_{t}(|V \xi|<C t)(x, I, 0)<C<1 .
$$

Proof. We introduce a function $g$ strictly decreasing, convex, from $[0, \infty[$ into $[0,1]$ equals to 1 in 0 and tending to 0 at infinity. We consider the function $F(\cdot): s \rightarrow$ $\hat{P}_{s}\left[g\left(\frac{|V \xi|}{t}\right)\right](x, I, 0)$. It has a derivative in 0 in $-C / t$ and a second derivative bounded by $C / t^{2}$. Moreover $F(0)=0$ and $F(C t)<C<1$ for some $t$. This shows the result.

\section{PROPOSITION 4.2.}

$\hat{P}_{t}[|V \xi|<\varepsilon](x, I, 0) \leq C_{p} \varepsilon^{p}$ for all $p$ uniformly in $|\xi|=1$. (We say in such a case that $\hat{P}_{t}[|V \xi|<\varepsilon](x, I, 0)=o\left(\varepsilon^{\infty}\right)$.)

Proof. We get for a big $C$,

$$
\hat{P}_{t}\left(\left|U^{-1}\right|>C\right](x, I, 0)=o\left(t^{\infty}\right) .
$$

In order to show that, we choose a positive function $g=0$ in a neighborhood of $I$ and equal to 1 far from $I$. We have, by using the parabolic equation satisfied by $\hat{P}_{t}$

$$
\frac{\partial^{r}}{\partial t^{r}} \bar{P}_{0}\left[g\left(U^{-1}\right)\right](x, I)=0
$$

for all $r$. Therefore the result.

The same result holds for $P_{t}(|\cdot-x|>C)(x)$. Moreover,

$$
\hat{P}_{t}[|V \xi|<\varepsilon](x, I, 0) \leq \hat{P}_{\varepsilon^{\alpha}}[|V \xi| \leq \varepsilon](x, I, 0)
$$

for $\alpha \leq 1$.

We slice $\left[0, \varepsilon^{\alpha}\right]$ in $\varepsilon^{-\beta}$ intervals with $\alpha+\beta<1$. By the previous lemma

$$
\sup _{|y-x|<C,\left|U^{-1}\right|<C} P_{\varepsilon^{\alpha+\beta}}[|V \xi| \leq \varepsilon](y, U, 0)<C<1 .
$$

We deduce by Markov property that

$$
\hat{P}_{\mathcal{E}^{\alpha}}[|V \xi|](x, I, 0)<C^{\mathcal{E}^{-\beta}}=o\left(\varepsilon^{\infty}\right) .
$$

Theorem 4.3. $\hat{P}_{t}\left[|V|^{-p}\right](x, I, 0)<\infty$ if $t>0$. 
Proof. We remark that

$$
\hat{P}_{t}\left(|V|^{p}\right](x, I, 0)<\infty
$$

for all $p>0$ (Proposition 3.3).

We choose $\varepsilon^{-\beta}$ points $\xi_{i}$ on the sphere of $R^{d}$ such that

$$
\begin{aligned}
& \hat{P}_{t}\left[\left|V^{-1}\right|>\varepsilon\right](x, I, 0) \\
& \quad \leq \sum \hat{P}\left[\left|V \xi_{i}\right|<\varepsilon\right](x, I, 0)+\hat{P}_{t}\left[|V|<\varepsilon^{-\gamma}\right](x, I, 0)=o\left(\varepsilon^{\infty}\right)
\end{aligned}
$$

for some suitable $\gamma>0$.

As a corollary, we get the following.

Theorem 4.4. Under (4.1), $f \rightarrow P_{t}[f](x)$ has a smooth density $p_{t}(x, y)$.

\section{Appendix}

Proof of Proposition 3.3. We work by induction on $k$. We choose for $k=1$ a smooth function $g(u)=0$ in $u=1$ and equal to $|u|$ when $u$ goes to infinity, with bounded derivatives. We get for two constant independent on $C$ that

$$
\begin{aligned}
& \left|\frac{\partial}{\partial t} P^{\mathrm{tot}, 1}\left[|u|^{2 r} \exp [-g(u) / C]\right](x)\right| \\
& \quad \leq K_{1}+K_{2} P^{\mathrm{tot}, 1}\left[|u|^{2 r} \exp [-g(u) / C]\right](x)
\end{aligned}
$$

for $r \in N$. The result follows by using the Gronwall lemma when $C \rightarrow \infty$.

We split up the equation giving $P\left[f\left(x_{k}\right)\right]$ in a equation giving a linear matrix and an equation which depends only on the previous terms as in Lemma 3.7. We get $P_{1}^{\text {tot }, k}\left[f\left(x_{k}\right)\right](\cdot, 0)=\hat{P}_{t}\left[f\left(\hat{u}_{k} \hat{v}_{k}\right)\right](\cdot, I, 0)$ as in Lemma 3.7. (We start from 0 in order to simplify the exposition.) We get $\hat{P}^{\text {tot }, k}\left[\left|\hat{u}_{k}\right|^{-p}\right](\cdot, I)<\infty$ because

$$
\begin{aligned}
& \left|\frac{\partial}{\partial t} \hat{P}^{\mathrm{tot}, k}\left[\left|\hat{u}_{k}\right|^{-2 r} \exp \left[-\hat{g}\left(\hat{u}_{k}\right) / C\right]\right](I)\right| \\
& \quad \leq K_{1}+K_{2} \hat{P}^{\mathrm{tot}, k}\left[\left|\hat{u}_{k}\right|^{-2 r} \exp \left[-\hat{g}\left[\hat{u}_{k}\right] / C\right]\right](I),
\end{aligned}
$$

where $\hat{g}\left(\hat{u}_{k}\right)$ is a function with bounded derivatives at infinity, bounded at infinity and equal to $\left|\hat{u}_{k}\right|^{-1}$ near 0 . The result arises by Gronwall lemma and making $C \rightarrow \infty$.

Moreover, if we do the change of variable $u_{k} \rightarrow u_{k}^{-1}$, we still get a semigroup governed by a generator of the same type. This shows that

$$
\hat{P}^{\mathrm{tot}, k}\left[\left|\hat{u}_{k}^{-1}\right|^{-2 r}\right](\cdot, I)<\infty .
$$

On the other hand,

$$
\left.\left.\frac{\partial}{\partial t} \hat{P}^{\mathrm{tot}, k}\left[\left|\hat{u}_{k}^{-1}\right|^{2 r} \bar{g}\left(\hat{u}_{k}\right) / C\right)\right](\cdot, I) \mid \leq K_{1}+K_{2} \hat{P}^{\mathrm{tot}, k}\left[\left|\hat{u}_{k}^{-1}\right|^{2 r} \bar{g}\left(\hat{u}_{k}\right) / C\right)\right](\cdot, I),
$$

where $\bar{g}\left(\hat{u}_{k}\right)$ is a smooth function with values in $[0,1]$ equal to 1 in 0 and equal in a neighborhood of infinity to $\left|\hat{u}_{k}^{-1}\right|^{-K}$ for a big $K$.

We use Gronwall lemma and by making $C \rightarrow \infty$ we deduce that $\hat{P}^{\text {tot }, k}\left[\left|\hat{u}_{k}^{-1}\right|^{2 r}\right](\cdot, I)<\infty$ and symmetrically that $\hat{P}^{\text {tot }, k}\left[\left|\hat{u}_{k}\right|^{2 r}\right](\cdot, I)<\infty$.

In order to estimate $\hat{P}^{\text {tot }, k}\left[\left|v_{k}\right|^{2 r}\right]$ we proceed in a similar but simpler way. 


\section{Acknowledgement}

The author would like to thank the Research Center of Theoretical Physics, Central Visayan Institute of Jagna, Bohol, Philippines for its kind hospitality where this work was done.

\section{References}

$\left[\mathrm{B}_{1}\right]$ Bismut $\mathrm{J}$ M, Martingales, the Malliavin calculus and hypoellipticity under general Hoermander's conditions, Zeit. Wahrscheinlichkeit 56 (1981) 469-505

$\left[\mathrm{B}_{2}\right]$ Bismut J M, The Atiyah-Singer theorems, a probabilistic approach I, J.Funct. Anal. 57 (1984) 56-99

[IW] Ikeda $\mathrm{N}$ and Watanabe $\mathrm{S}$, Stochastic differential equations and diffusion processes (North Holland) (1981)

$\left[\mathrm{L}_{1}\right]$ Léandre R, Extension du théorème de Hoermander à divers processus de sauts, Thèse (Université de Franche-Comté) (1984)

$\left[\mathrm{L}_{2}\right]$ Léandre R, Applications quantitatives et géométriques du Calcul de Malliavin, in: Séminaire Franco-Japonais (eds) M Métivier and S Watanabe, Lect. Notes Math. 1322 (1988) pp. 109-134, English translation: Geometry of random motion (eds) R Durrett and M Pinsky, Cont. Maths. 73 (1988) 173-196

$\left[\mathrm{L}_{3}\right]$ Léandre R, Sur le théorème d'Atiyah-Singer, Probab. Theory. Rel. Fields 80 (1988) $119-137$

[ $\left.\mathrm{L}_{4}\right]$ Léandre R, Régularité de processus de sauts dégénérés (II), Ann. Inst. Henri Poincaré 24 (1988) 209-236

[Ma $]$ Malliavin P, Stochastic calculus of variations and hypoelliptic operators, in: Stochastic analysis (ed.) K Itô (Kinokuniya) (1978) pp. 155-263

$\left[\mathrm{Ma}_{2}\right]$ Malliavin $\mathrm{P}, C^{k}$ hypoellipticity with degeneracy, in: Stochastic Analysis (eds) A Friedmann and M Pinsky (Acad. Press) (1978) pp. 199-214

$\left[\mathrm{Ma}_{3}\right]$ Malliavin P, Stochastic Analysis (Springer) (1997)

[Me] Meyer P A, Le Calcul de Malliavin et un peu de pédagogie. R.C.P 34. (Strasbourg: Pub. Univ.) (1984)

[No] Norris J, Simplified Malliavin calculus, in: Séminaire de Probabilités XX (eds) J Azéma and M Yor, Lect. Notes. Math. (1986) pp. 101-131

[Nu] Nualart D, The Malliavin calculus and related topics (Springer) (1995)

[St] Stroock D, Some applications of stochastic calculus to partial differential equations, in: Ecole d'Été de Saint-Flour X (ed.) P Hennequin, Lect. Notes Math. 876 (1983) pp. 267-382 